\title{
Public Opinion, Propaganda, Conspiracy Theories, and Education: An Interview with Dr. Andrew M. Clark
}

\section{Dr. Yubraj Aryal}

Dr. Andrew M. Clark, Associate Director of the Center for Research on Teaching and Learning Excellence (CRTLE) and Associate Professor in the Department of Communication at UTA, was interviewed by Global Insight Executive Editor, Dr. Yubraj Aryal.

Y. A.: By drawing on your many decades of combined experience with communication students, observing their maturation into communicative practice in academic contexts, could you tell me some of the essential values of communication education?

A. C.: We offer our students opportunities in Communication Studies and in the Mass Communication areas such as Advertising, Public Relations, Broadcasting, Journalism and Communication Technology. What we do is empower our students to use a variety of tools to both evaluate effective communicative practices and to engage in effective communicative practices in many different contexts and across many different platforms. We help our students to understand their audience and to develop robust content that reaches that audience on a variety of levels. Our students go into careers such as sales, training and development, research, journalism, reporting, videography and editing, web design, social media marketing and so much more. I hope that as they embark on their careers, they do so being proficient with the needed technical skills, but also having developed valuable life skills that make them not only better communicators, but better people.

Y. A.: After the recent explosion of conspiracy theories, what kind of tension are you experiencing in the teaching of the liberal arts education and research?

A. C.: There are plenty of hot button issues and plenty of opinions about those issues. On a college campus they can be about major issues such as conspiracy theories about COVID and about vaccinations, but there are also campus conspiracy theories among students such as about parking on campus. They are obviously extremes on a spectrum, but the lessons are similar. When I teach broadcast news my concern is that students be able to produce a news story that is balanced and enables both perspectives. This involves research, writing, and editing among other skills. I know that I have students in my class that have strong opinions and the challenge is to get 
DR. ANDREW M. CLARK

them to write in such a way that they're reporting the facts of the story without injecting their own opinion into it. It is hard to put together a story when you feel strongly about an issue. It is hard to ensure that differing sides of the equation are represented and where the goal is not to produce something that aligns with your ideas, but that lets the viewer make their decision based on the information you present. These are life lessons as well.

Y. A.: We're living in an age where public opinions have completely swerved either too far-right or too farleft. How do you want to encourage our undergraduates to form only evidence-based independent opinions/ knowledge about the world we live in?

A. C.: It's hard because we all have opinions shaped by our background, our culture, our beliefs, and so on. What I think the challenge is, is to understand each other and instead of judging each other because you think differently from me but try and understand why you think the way that you do. We label people and if you believe one thing you're labeled this and if you believe something else you're labeled that, and labeling can be very destructive. It also strips us of our individuality and instead lumps us as part of a group. It takes time to learn about someone and to try and understand them, even a little. I think as educators we can develop lessons and assignments that teach how to sort fact from fiction, and how to evaluate a message. However, I think even more important is to find ways to teach respect and to model that behavior ourselves by what we say and do. We must develop lessons that give students the opportunity to work with people different from themselves and encourage respect for other people even if we do not always agree.

Y. A.: Since you also co-direct the CRTLE at UTA, how does your Center benefit our undergraduates?

A. C.: CRTLE's primary focus is on faculty with some programs for graduate students. However, undergraduate students benefit because, as we help faculty develop and become successful in their teaching, then students reap the benefits in the classes that they take. In short, I hope that faculty success leads to student success. 